\title{
Proteoform analysis to fulfill unmet clinical needs and reach global standardization of protein measurands in clinical chemistry proteomics
}

Yuri E.M. van der Burgt ${ }^{1,2}$ and Christa M. Cobbaert ${ }^{1}$

${ }^{1}$ Department of Clinical Chemistry and Laboratory Medicine

${ }^{2}$ Center for Proteomics and Metabolomics

Leiden University Medical Center (LUMC), PO Box 9600, 2300 RC, Leiden, the Netherlands.

\author{
Corresponding Author: \\ Yuri E.M. van der Burgt \\ Leiden University Medical Center (LUMC) \\ Department of Clinical Chemistry and Laboratory Medicine \\ PO Box 9600, 2300 RC, Leiden, The Netherlands \\ Email: y.e.m.van_der_burgt@lumc.nl
}

\section{For a special issue:}

in Clinics in Laboratory Medicine on Clinical Pathology, edited by Dr. Geza S. Bodor

\section{KEY WORDS:}

Proteomics; Mass spectrometry; Clinical chemistry proteomics; Measurand; Protein quantification; Proteoforms; Protein glycosylation; Metrological traceability. 


\section{SYNOPSIS}

In clinical testing of protein markers structure variants of the measurand are often not taken into account. This heterogeneous character of protein measurands in immunoassays often renders test standardization impossible. Consequently, test results from different methods can lead to under- or overdiagnosis and thus under- or overtreatment of patients. The systematic structural analysis of protein isoforms has been coined proteoform profiling and is performed through mass spectrometry-based proteomics strategies. Knowledge on proteoforms allows refining existing unimarker tests and moreover has great potential to contribute to the urgent need for new tests to predict prognosis and severity of diseases. 


\section{INTRODUCTION}

\section{Protein biomarkers in precision medicine}

In the pursuit of individualized patient treatment biomarkers play a crucial role. Although genomic and transcriptomic analyses are of great value in the clinic, the complexity of the human body largely arises from variations in protein identities and quantities. In basic research mass spectrometry (MS)-based proteomics has greatly contributed to an understanding of cellular functions at a molecular level [1-3]. Also, large-scale exploratory efforts have been applied on retrospective studies of (large) clinical cohorts of body fluids such as plasma or serum samples searching for novel biomarkers. Hitherto the number of new protein markers that made it from MS-based proteomics into the clinic is very limited [4]. Rather than having a technological origin, key-reasons for this translation-lag are the use of invalid samples, lack of thoughtful study designs, silo-thinking of the stakeholders involved and lack of appropriate test evaluation and adequate test standardization [5]. In clinical laboratories proteins in body fluids are routinely tested for diagnostic and prognostic purposes, as well as for therapy monitoring. It is however widely acknowledged that there is room for improvement with regard to sensitivity and specificity levels of current medical tests [6]. Moreover, clinically effective disease-specific tests that support diagnoses at an early and curable stage are still lacking for a wide variety of diseases. Aiming and searching for novel (protein) biomarkers should start by defining specific unmet clinical needs with the clinicians according to test evaluation checklist [5,7]. Interestingly, post-translational modifications (PTMs) on proteins have often not been taken into account because of technical challenges and the increased complexity of the resulting data. PTMs on existing protein biomarkers provide an additional structural layer to quantitative levels of individual 
proteins with potential for patient stratification. Here, the often ignored presence of PTMs in protein tests and their implication for clinical chemistry proteomics (CCP) will be discussed.

\section{What is the "proteoform hypothesis"?}

Comprehensive proteome information contributes to a systems-level understanding of human biology and thus disease [3,8]. Recently, interest in protein PTMs with regard to biological and clinical relevance has emerged as an additional layer in proteomics, and has been coined as proteoform analysis $[9,10]$. The term proteoform was proposed in 2012 "to designate all of the different molecular forms in which the protein product of a single gene can be found" and was swiftly adapted by the proteomics community [11]. Although protein isoforms and PTMs are long-known from gel electrophoresis and chromatography, in the early days of proteomics identifications (IDs) were based on so-called peptide (mass) fingerprints with the aim to determine any protein product from a single gene (and not each). An inherent effect was that the focus switched to the number of protein IDs and that isoforms were not considered anymore [12]. Since the beginning of this century, with the availability of the human genome, MS-based peptide sequencing has been optimized and turned into the method of choice for bottom-up proteomics (i.e. identification of proteotypic peptides after digestion with a protease). Instrument development benefitted from the large growth in proteomics applications MS and was further developed with regard to speed and userfriendliness. Moreover, these innovations led to ultrahigh resolution platforms such as orbitraps $^{\mathrm{TM}}$ and Fourier ion cyclotron resonance mass spectrometers that provide improved mass precision and accuracy and consequently yield more confident identifications [13]. Furthermore, these high-end platforms allow for a mass measurement of proteins in their intact form and offer a wide range of fragmentation techniques. So-called top-down proteomics studies have converged into a renewed interest in protein isoforms, i.e. 
proteoforms, and have opened an exciting field within clinical MS [10]. Proteoforms arise from a single gene from changes due to genetic variations, alternatively spliced RNA transcripts and PTMs (see Figure 1). An example of the latter involves histone modifications that in a biological context are referred to as epiproteomic signatures $[14,15]$. Note that structurally related protein forms from different genes are not grouped together to ensure that the proteoform terminology remains compatible with a gene-centric approach [16].

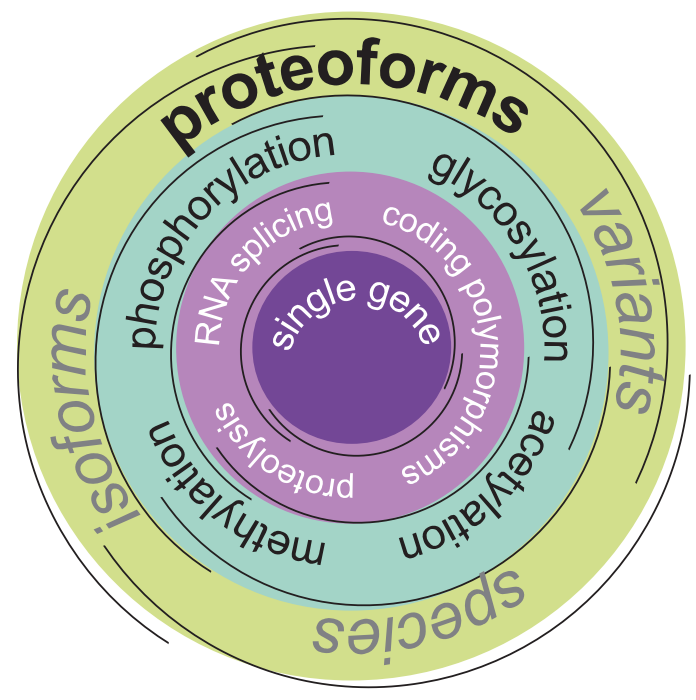

\section{Figure 1.}

Protein structural variants that originate from a single gene as a result of transcriptional processing and/or diverse post-translational modifications. Proteoforms can furthermore arise from changes in the gene itself (allelic variants from coding polymorphisms or mutation). The terms protein isoforms, variants and species are greyed-out since these are ambiguous and do not accurately reflect the measurand of interest.

Soon after the first comprehensive proteoform analyses were performed and shown to be beneficial, the same group of Kelleher postulated a corresponding hypothesis, namely that 
"intact proteoforms represent a powerful class of molecules for use as biomarkers of disease states" [17]. Here the term powerful should be interpreted as "the ability to detect a true difference between two or more populations when such a difference is present". The hypothesis refers to for example "detection of the presence or absence of cancers, the onset of disease, the classification of cell types or the differentiation of two or more biological states". Indeed proteoform analysis provides a new layer of information that complements data related to transcription, translation and post-translational events that underlie complex phenotypes [18]. Genotyping and transcriptomic analysis are not telling the full story, for example it was reported that mRNA abundances only weakly correlate with protein expression levels [19]. However, whether or not the proteoform hypothesis holds promise for clinical purposes partly depends on technological robustness and moreover has to be determined from measurements in large patient cohorts. In addition, methodological challenges need to be overcome and requirements to be met to show that proteoform-resolved data truly improves patient stratification.

\section{Clinical chemistry proteomics and metrological traceability}

Protein quantification in medical laboratories is mostly performed with commercially available, CE-marked (Conformité Européenne, i.e. European conformity) antibody-based immunoassays. For personalized patient care, accuracy of test results is essential and for correct interpretation matching of reference values and/or decision limits is needed. In order to achieve standardized test results, metrological traceability is defined as: 'property of a measurement result whereby the result can be related to a reference through a documented unbroken chain of calibrations, each contributing to the measurement uncertainty' [20]. Ultimate test standardization is reached when the entire reference system and metrological traceability chain are in place. This is not trivial since the measured proteins often have a 
heterogeneous character (proteoforms!). Moreover, commutable and value-assigned reference materials are often not available, and internationally recognized reference measurement procedures are mostly lacking [21]. As a result, non-comparable results are reported between laboratories, for example in the case of thyroglobulin [4,22]. MS-based technologies are the method of choice for tackling these issues and recently steps towards harmonized MS-based measurements have been reported [23-25]. This approach is also used by working groups and committees of the International Federation of Clinical Chemistry (IFCC) for standardization of protein measurands (www.ifcc.org/ifcc-scientific-division/). Such MS-based targeted peptide measurements for protein biomarker quantification require analytical quality that is in agreement with their intended clinical use. A test that is fit-for-purpose should meet predefined analytical performance goals for bias and imprecision based on the biological variation(s) of the analyte(s) within and between individuals. Quantitative CCP aims for transfer of MS-based methodologies into a routine assay including metrological traceability with suitable calibrators and certified reference materials [26]. In addition, aforementioned ultrahigh resolution MS platforms allow detailed structural characterization of various proteoforms in the heterogeneous mixture of analytes, whereas in a medical test these may be interpreted as a single protein, i.e. measurand. In an immunoassay all proteoforms that are recognized by the antibody, either biologically active or inactive, will be captured and as a result a summarized protein quantity is obtained. Thus, in case the measurand is actually a mixture and is therefore undefined, standardization of immunoassays will be difficult to achieve. Proteoform analysis will not necessarily replace such immunoassays, but certainly complement to standardization efforts. Successful implementation of the traceability concept for quantitative MS-based CCP methods, along with unambiguous definitions of the measurand, should enable the development of traceable reference intervals and/or decision limits. Due to the heterogeneous character of the protein analyte in a medical test the 
measurand can differ in the immunoassay and the CCP method and both require their own reference ranges and/or decision limits. With an increasing number of laboratories involved in quantitative MS-based CCP methods, EQA- and professional clinical chemistry organizations have started collaborations and joint projects to determine traceable reference intervals and/or decision limits for protein measurands [27-30]. In addition these collaborations will deliver a template for further development of these MS-based methods into validated assays and finally clinical tests. 


\section{STRATEGIES FOR PROTEOFORM ANALYSIS}

\section{Proteoform sampling, separation and identification}

An important aspect of any protein quantification experiment, but nevertheless often overlooked, involves the pre-analysis part. Important considerations include specimen collection and transportation, and sample handling and storage, since proteins are not inert and sample degradation may be biased for certain proteoforms or yield "new" proteoforms (for example due to oxidation or deamidation). With regard to sample preparation it is stressed that protein standards are often provided in buffer that are not compatible with MS analysis and that in these cases purifications may be needed to enable structural characterization studies. Obviously, an up-front proteoform separation simplifies sequential structure analysis, however chromatography of intact proteins is more difficult than for any other biomolecule [31]. The first proteoform experiments were mostly based on offline purified proteins, in which the mass analyzer was used as the separation device [32]. Such an approach remains valid, however it is not attractive with regard to current high-throughput requirements including well-defined reproducibility and robustness. Fortunately, progress has been made and nowadays online strategies have become available that provide proteoform separations. The two core technologies that are used involve liquid chromatography (LC) and capillary zone electrophoresis (CZE). Proteoform identifications are performed through bottom-up as well as top-down approaches [33]. The identification and quantification of proteins is routinely performed via MS-based bottom-up proteomics (also referred to as shotgun or peptide-centric), in which proteins are proteolytically cleaved (digested) into peptides that are most commonly separated by online LC and identified using tandem MS. With the development and introduction of quantitative CCP tests, the analytical specificity is enhanced and moreover, any clinically relevant proteoform can be detected and quantified. 
Essential elements for proper quantitative CCP standardization including metrological traceability are defining the measurands, selecting suitable proteotypic peptides, preparing labeled internal standards, optimizing proteolysis aiming for equimolarity between protein and peptide measurement, and calibration using well-defined internal standards and/or external calibrators. In top-down proteomics intact proteins are analyzed and characterized without digestion using ultrahigh resolution MS platforms such as mentioned in the introduction of this paper. Top-down MS provides information on the intact protein mass and allows for identification of novel proteoforms, in-depth sequence characterization, and quantitation of disease-associated PTMs. Top-down proteomics has given additional momentum for global and comprehensive analysis of proteoforms in existing (approved) protein markers as an additional layer of structural information.

\section{Protein glycosylation}

One of the most common (and arguably complex) PTMs involves protein glycosylation. The vast majority of membrane and secreted proteins are known or predicted to be $\mathrm{N}$ - and Oglycosylated. Glycoproteins represent key molecules in many important biological processes such as cell adhesion, endocytosis, receptor activation, signal transduction, molecular trafficking, and clearance, as well as in diseases, including cancer. In MS-based proteomics studies this PTM has often not been considered because of various reasons. First of all, whereas for example methyl-, phosphate- and acetyl-groups can be accommodated in database searches by an exactly defined mass difference, glycans are structurally diverse with different sizes (monomeric to oligomeric), and moreover can have isobaric identities. Consequently, glycan identifications require additional experiments, such as characterization of enzymatically released $\mathrm{N}$-glycans or even orthogonal analytical strategies or instrumentation (e.g. nuclear magnetic resonance spectroscopy). Secondly, to study the 
clinical relevance of protein glycosylation the applied MS-based strategies require robust and high-throughput (HT) platforms that have only recently become available [34]. HT protein glycosylation studies are a type of proteoform analysis, and consequently this term is interchangeably used with the long-used glycoform [35]. In-depth approaches to determine site-specific protein glycosylation have become indispensable tools for functional analyses of these complex biomolecules. Protein glycosylation is known to change during disease and potentially offers a rich source of biomarkers. Discovery is either pursued on the level of a single protein by mapping all its proteoforms, or by HT glycomics approaches. This first (single protein) approach is of interest for clinical chemistry purposes, since it can involve detailed characterization of an existing protein biomarker (as was pointed out earlier in the Introduction section). An brief example is discussed in the Applications section. With regard to the latter approach the so-called total serum N-glycome (TSNG) comprises the N-glycans from all serum proteins, which are to a large extent liver- (acute-phase proteins) and plasma cell-derived (antibodies). Recent developments in MS-based HT glycosylation analysis have provided the opportunity to acquire information on TSNG N-glycan complexity, antennarity, galactosylation, fucosylation, as well as on the presence and linkage of sialic acids $(\alpha 2,6-$ versus $\alpha 2,3-$ linkage). Although these TSNG-studies have yielded interesting glycan biomarker candidates for various diseases, exploratory efforts fall outside the scope of this review and will not be further discussed. 


\section{APPLICATIONS IN CLINICAL CHEMISTRY PROTEOMICS}

The benefits of clinical MS for protein quantitation have been pointed out and are increasingly acknowledged by clinical chemists [27]. As mentioned here, one of the major advantages involves test standardization according to ISO 17511:2003 so that test results become traceable to standards of higher order and comparable among hospital labs. Test result comparability is essential in this era of electronic health records and free movement of patients across health institutions [21,25]. A second important driver for implementing MSbased assays in a medical laboratory is the ability to multiplex quantification of various proteins [36,37]. This is in line with current MS-based proteomics strategies that provide extensive lists of protein identities and quantities in clinical samples such as tissue or body fluids. Here, the quest for a single biomarker in either retrospective or prospective study cohorts has turned into an approach in which protein signatures are aimed for, or even full proteomes i.e. proteotypes, are reported [38]. However, the goal to fully describe a proteome is only partly met when listing large numbers of protein IDs. It has become clear that detailed structural knowledge on proteoforms has become a crucial aspect in many studies that involve proteome analysis [39-41]. The same holds true for the in-depth analysis of the heterogeneous character of protein measurands.

Multiple protein standardization efforts are carried out by the Scientific Division of the IFCC, wherein structural heterogeneity of the measurand needs consideration (see also Introduction). Although the biological and/or clinical importance of specific proteoforms has been demonstrated for certain proteins in the context of disease, it is also noted that validation of the clinical relevance of proteoform profiling requires further studies with larger patient cohorts. Examples of currently applied protein biomarkers that actually consist of a mixture 
of proteoforms are transferrin, apolipoproteins and prostate-specific antigen (PSA). In the case of transferrin, it has been shown that abnormal transferrin glycosylation is known as a biochemical marker for the congenital disorders of glycosylation (CDG), a group of inherited metabolic disorders with defects in protein and lipid glycosylation [42-44]. Similarly, glycosylation profiling of transferrin is the cornerstone of the test for alcohol abuse (i.e. carbohydrate-deficient transferrin) $[45,46]$. In the case of apolipoproteins, these are constituents of lipoprotein particles and furthermore key-players in lipoprotein metabolism. Different glycoforms, i.e. proteoforms, of apolipoproteins have an effect on lipid metabolism $[47,48]$. In our laboratory, we have developed a quantitative CCP test for multiplexed measurement of apolipoproteins that has been applied in various studies on clinical cohorts for over three years, such as on cardiovascular risk assessment $[49,50]$. It should be noted that in these quantitative CCP measurements of apolipoprotein C-III all proteoforms with known as well as newly reported O-glycans (with fucosylation) are summarized into one value (see Figure 2) [51]. It has been reported that sialylation levels of apolipoprotein C-III in serum associate with improved lipids for type 2 diabetes and prediabetes [52]. Proteoforms of apolipoproteins C-I and C-II have also been reported [53]. Such different proteoforms can potentially be included in our lab-developed test [37]. The role of newly discovered apolipoprotein C-III fucosylation needs further investigation. As a side remark, the here mentioned quantitative $\mathrm{CCP}$ test also includes apolipoprotein $\mathrm{E}$ phenotyping derived from genetic variants within the patient cohorts. 


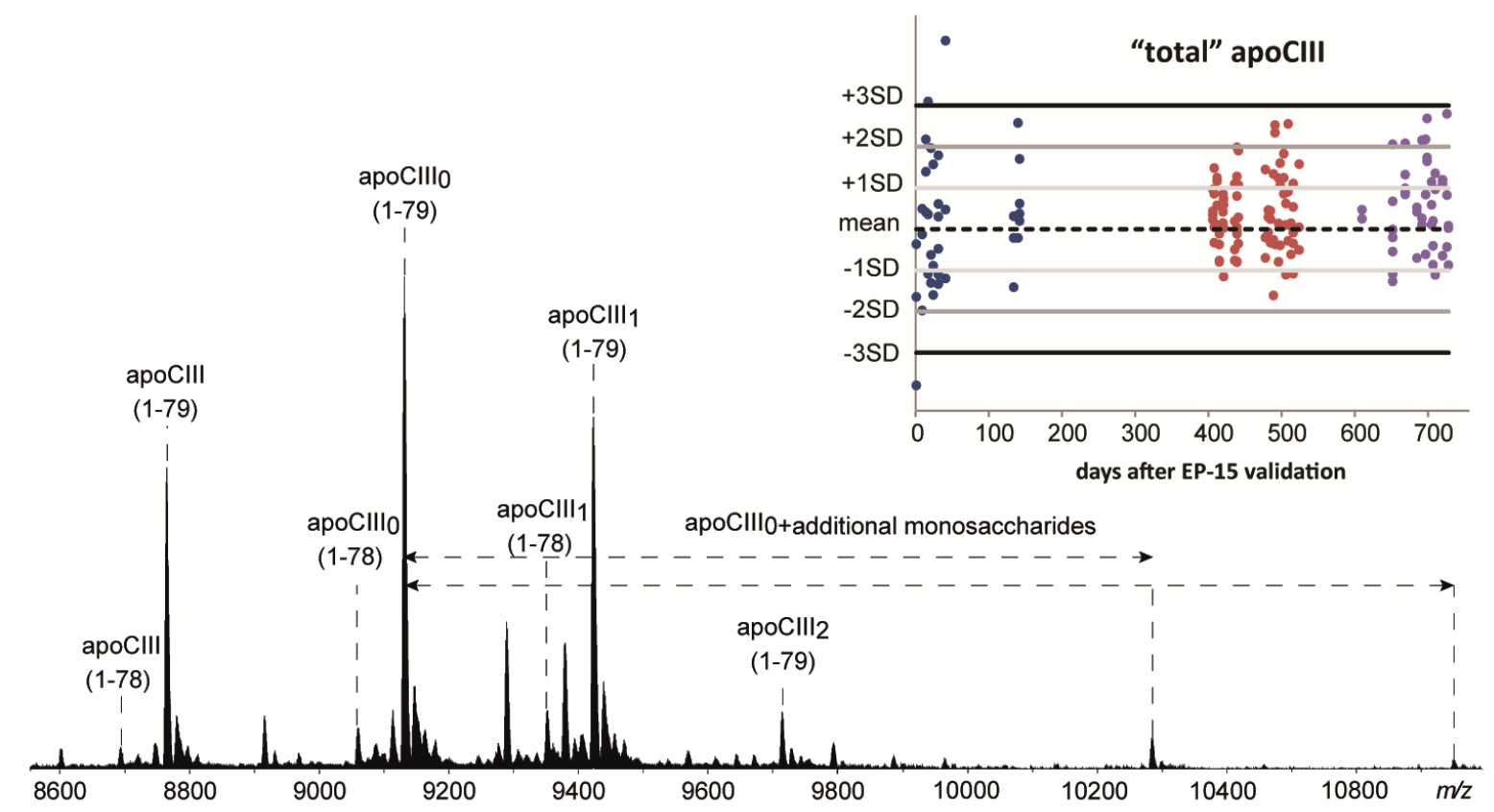

\section{Figure 2}

Intact proteoforms of apolipoprotein C-III in an ultrahigh resolution MALDI mass spectrum (mass scale on the $\mathrm{x}$-axis, nine proteoforms are assigned). Proteoforms originate from protein O-glycosylation (subscripts $0,1,2$ indicate the number of sialic acids on the glycan and "monosaccharides" refer to fucosylation and glycan chain elongation) or from differences in protein size/length (numbers between parentheses reflect the amino acid sequence of apoCIII) [51]. In the inset a Levey-Jennings chart is shown of apoCIII quantities in a quality control sample measured over more than 700 days (on the $\mathrm{x}$-axis) [50]. On the y-axis, each value (determined via quantitative CCP) represents the sum of all proteoforms, i.e. total apoCIII, whereas in the ultrahigh resolution mass spectrum each apoCIII proteotype is observed as a separate signal.

A third example of a routinely applied protein biomarker that consists of proteoforms is PSA. Although the protein concentration of PSA in serum is an FDA-approved method for early detection of prostate cancer (PCa), its sensitivity is rather poor [54]. Currently, elevated PSA values (above $3 \mathrm{ng} / \mathrm{mL}$ or $4 \mathrm{ng} / \mathrm{mL}$ ) are often followed by additional investigations such as 
digital rectal examination (DRE), magnetic resonance imaging (MRI) and/or prostate biopsy [54,55]. Moreover, the PSA test also lacks specificity since benign prostate hyperplasia or prostatitis can also result in elevated levels of PSA. Aiming for an improved PSA test, glycosylation of PSA has recently been described based on glycopeptide analysis, in which a total of $67 \mathrm{~N}$-glycopeptides (proteoforms!) were identified [56]. Here it was concluded that PSA proteoform profiling might be a promising tool for the determination of potential glycomic biomarkers for the differentiation between aggressive PCa, indolent PCa and benign prostate hyperplasia in larger cohort studies. 


\section{FUTURE PERSPECTIVES}

Proteins in body fluids are routinely tested in clinical laboratories for diagnostic, prognostic, disease classification and monitoring purposes [57]. Yet, facing the large number of unmet clinical needs (e.g. so far no tests are available for specific diseases like chronic obstructive pulmonary disease and acute kidney injury) and insufficient clinical performance of contemporary tests, there is room for improvement. MS-based methods in general and quantitative CCP specifically, will complement "total" protein read-outs obtained through immunoassays. In order to get this into place, a holistic and sustainable approach for demonstrating added value of quantitative CCP tests is needed, taking into account the concept of metrological traceability according to ISO 17511:2003 [21]. The use of structured frameworks for test evaluation, such as the framework of the European Federation of Laboratory Medicine, guarantees that only tests that are fit-for-clinical purpose are developed and implemented [5]. Although some routinely measured proteins are successfully standardized at the global level, most protein tests are not yet standardized [30]. To this end we pursue an integral approach to identify new protein markers as well as to measure existing markers by MS. In this context it is stressed that biomarker translation and method transfer go hand-in-hand.

With this paper we aim to make laboratory researchers, clinicians as well as in-vitro diagnostic (IVD)-manufacturers aware of the heterogeneous character of measurands in medical tests. To this end, knowledge on the identity and presence of various proteoforms in both reference samples and real-life patient samples is crucial both for a better understanding of the pathophysiology of disease for patient stratification and for enabling standardization according to the metrological traceability concept described in ISO 17511:2003. Proteoform 
analysis provides a stratification layer additional to quantitative levels of individual proteins or protein panels that already serve as biomarkers. The measurement of specific proteoforms may render MS-based strategies feasible as an add-on test to the corresponding protein quantification test. Proteoform profiling provides a golden opportunity for fulfilling unmet clinical needs in this era of precision medicine, and for standardizing protein measurands at the molecular level in order to make test results comparable worldwide. Their analysis is of interest for the rapidly growing number of laboratory specialists and IVD-manufacturers that acknowledge the potential of clinical MS and have share their experience within the emerging community Mass Spectrometry Applications in the Clinical Laboratory (MSACL). 


\section{REFERENCES}

[1] Aebersold R, Mann M. Mass-spectrometric exploration of proteome structure and function. Nature 2016;537:347-55.

[2] Altelaar AFM, Munoz J, Heck AJR. Nat Rev Genet 2013;14:35-48.

[3] Hughes CS, Foehr S, Garfield DA, et al. Ultrasensitive proteome analysis using paramagnetic bead technology. Mol Syst Biol 2014;10:757.

[4] Anderson L. Within sight of a rational pipeline for development of protein diagnostics.

Clin Chem 2012;58:28-30.

[5] Monaghan PJ, Lord SJ, St John A, et al. Test Evaluation Working Group of the European Federation of Clinical Chemistry and Laboratory Medicine. Biomarker development targeting unmet clinical needs. Clin Chim Acta 2016; 460:211-9.

[6] Hoofnagle AN, Wener MH. The fundamental flaws of immunoassays and potential solutions using tandem mass spectrometry. J Immunol Methods 2009;347:3-11.

[7] Horvath AR, Lord SJ, St John A, et al. Test Evaluation Working Group of the European Federation of Clinical Chemistry Laboratory Medicine. From biomarkers to medical tests: the changing landscape of test evaluation. Clin Chim Acta 2014;427:49-57.

[8] Geyer PE, Kulak NA, Pichler G, et al. Plasma Proteome Profiling to Assess Human Health and Disease. Cell Syst 2016;2:185-95.

[9] Smith LM, Kelleher NL. Consortium for Top Down Proteomics: Proteoform: a single term describing protein complexity. Nat Methods 2013;10:186-187.

[10] Savaryn JP, Catherman AD, Thomas PM, et al. The emergence of top-down proteomics in clinical research. Genome Med 2013;5:53.

[11] Kelleher N. A cell-based approach to the human proteome project. J Am Soc Mass Spectrom 2012;23:1617-1624.

[12] Nilsson T, Mann M, Aebersold R, et al. Mass spectrometry in high-throughput proteomics: ready for the big time. Nat Methods 2010;7:681-685.

[13] Mann M, Kelleher NL. Precision proteomics: the case for high resolution and high mass accuracy. Proc Natl Acad Sci U S A 2008;105:18132-8.

[14] Dai B, Rasmussen TP. Global epiproteomic signatures distinguish embryonic stem cells from differentiated cells. Stem Cells 2007; 25:2567-2574.

[15] Young NL, DiMaggio PA, Plazas-Mayorca MD et al. High Throughput Characterization of Combinatorial Histone Codes. Mol Cell Prot 2009;8:2266-2284.

[16] The UniProt Consortium. Nucleic Acids Res. 2012; 40:D71-D75.

[17] Kelleher NL, Thomas PM, Ntai I, et al. Deep and quantitative top-down proteomics in clinical and translational research. Expert Rev Proteomics 2014;11:649-51.

[18] Aebersold R, Agar JN, Amster IJ, et al. How many human proteoforms are there? Nat Chem Biol 2018; 14:206-214.

[19] Khan Z, Ford MJ, Cusanovich DA, et al. Primate transcript and protein expression levels evolve under compensatory selection pressures. Science 2013;342:1100-4 
[20] JCGM200. International vocabulary of metrology - Basic and general concepts and associated terms. 2012. Available at: http://www.bipm.org/utils/common/documents/jcgm/JCGM_200_2012.pdf. Accessed at 20 April, 2018.

[21] Smit N, Van Den Broek I, Romijn FP, et al. Quality requirements for quantitative clinical chemistry proteomics. Translational Proteomics 2014;2:1-13.

[22] Preissner CM, O'Kane DJ, Singh RJ, et al. Phantoms in the assay tube: heterophile antibody interferences in serum thyroglobulin assays. J Clin Endocrinol Metab 2003;88:3069-3074.

[23] Carr SA, Abbatiello SE, Ackermann BL, et al. Targeted peptide measurements in biology and medicine: best practices for mass spectrometry-based assay development using a fit-for-purpose approach. Mol Cell Proteomics 2014;13:907-17.

[24] Annesley TM, Cooks RG, Herold DA, et al. Clinical Mass Spectrometry-Achieving Prominence in Laboratory Medicine. Clin Chem 2016;62:1-3.

[25] Netzel BC, Grant RP, Hoofnagle AN, et al. First Steps toward Harmonization of LC-MS/MS Thyroglobulin Assays. Clin Chem 2016;62:297-9.

[26] Lehmann S, Poinot P, Tiers L, et al. From“Clinical Proteomics" to "Clinical Chemistry Proteomics": considerations using quantitative mass-spectrometry as a model approach. Clin Chem Lab Med 2012;50:23542.

[27] Lehmann S, Brede C, Lescuyer P, et al. Clinical mass spectrometry proteomics (cMSP) for medical laboratory: What does the future hold?. Clin Chim Acta 2016;(16)30246-30247.

[28] Sandberg S, Fraser CG, Horvath AR, et al. Defining analytical performance specifications: consensus statement from the 1st strategic conference of the European Federation of Clinical Chemistry and Laboratory Medicine. Clin Chem Lab Med 2015;53:833-5.

[29] Dittrich J, Adam M, Maas H, et al. Targeted On-line SPE-LC-MS/MS Assay for the Quantitation of 12 Apolipoproteins from Human Blood. Proteomics 2018;18(3-4).

[30] Merlini G1, Blirup-Jensen S, Johnson AM, et al. IFCC Committee on Plasma Proteins (C-PP). Standardizing plasma protein measurements worldwide: a challenging enterprise. Clin Chem Lab Med 2010;48:1567-1575

[31] Chen B, Brown KA, Lin Z, et al. Top-Down Proteomics: Ready for Prime Time? Anal Chem 2018;90:110127.

[32] Kelleher NL, Lin HY, Valaskovic GA, et al. Top down versus bottom up protein characterization by tandem high-resolution mass spectrometry. J Am Soc Mass Spectrom 1999;121:806-812.

[33] Bogdanov B, Smith RD. Proteomics by FTICR mass spectrometry: top down and bottom up. Mass Spectrom Rev 2005;24:168-200.

[34] Bladergroen MR, Derks RJ, Nicolardi S, et al. Standardized and automated solid-phase extraction procedures for high-throughput proteomics of body fluids. J Proteom 2012;77:144-153.

[35] Dell A, Morris HR. Glycoprotein structure determination by mass spectrometry. Science 2001;291:2351-6. [36] Van Den Broek I, Nouta J, Razavi M, et al. Quantification of serum apolipoproteins A-I and B-100 in clinical samples using an automated SISCAPA-MALDI-TOF-MS workflow. Methods 2015;81:74-85. 
[37] Van Den Broek I, Romijn FP, Nouta J, et al. Automated multiplex LCMS/MS assay for quantifying serum apolipoproteins A-I, B, C-I, C-II, C-III, and E with qualitative apolipoprotein E phenotyping. Clin Chem 2016;62:188-197.

[38] Röst HL, Malmström L, Aebersold R. Reproducible quantitative proteotype data matrices for systems biology. Mol Biol Cell 2015; 26:3926-3931.

[39] Nicolardi S, Bladergroen MR, Deelder AM, et al. SPE-MALDI Profiling of Serum Peptides and Proteins by Ultrahigh Resolution FTICR-MS. Chromatographia 2014; DOI 10.1007/s10337-014-2812-8

[40] Trenchevska O, Nelson RW, Nedelkov D. Mass spectrometric immunoassays for discovery, screening and quantification of clinically relevant proteoforms. Bioanalysis. 2016;8:1623-1633.

[41] Nedelkov D. Human proteoforms as new targets for clinical mass spectrometry protein tests. Expert Rev Proteomics 2017;14:691-699.

[42] van Scherpenzeel M, Steenbergen G, Morava E, et al. High-resolution mass spectrometry glycoprofiling of intact transferrin for diagnosis and subtype identification in the congenital disorders of glycosylation. Transl Res 2015;166:639-649.

[43] Marklova E, Albahri Z. Screening and diagnosis of congenital disorders of glycosylation. Clin Chim Acta 2007;385:6-20.

[44] Hoshi K, Matsumoto Y, Ito H, et al. A unique glycan-isoform of transferrin in cerebrospinal fluid: A potential diagnostic marker for neurological diseases. Biochim Biophys Acta 2017;1861:2473-2478.

[45] Allen J, Litten R, Anton R, et al. Carbohydrate-deficient transferrin as a measure of immoderate drinking: remaining issues. Alcohol Clin Exp Res 1994;18:799-812.

[46] Sillanaukee P, Olsson U. Improved diagnostic classification of alcohol abusers by combining carbohydratedeficient transferrin and gamma-glutamyltransferase. Clin Chem 2001;47:681-5.

[47] van den Boogert MAW, Rader DJ, Holleboom AG. New insights into the role of glycosylation in lipoprotein metabolism. Curr Opin Lipidol 2017;28:502-506.

[48] Yen-Nicolaÿ S, Boursier C, Rio M, et al. MALDI-TOF MS applied to apoC-III glycoforms of patients with congenital disorders affecting O-glycosylation. Comparison with two-dimensional electrophoresis. Proteomics Clin Appl 2015;9:787-93.

[49] Hermans MPJ, Bodde MC, Jukema JW, et al. Low levels of apolipoprotein-CII in normotriglyceridemic patients with very premature coronary artery disease: observations from the MISSION! Intervention study. J Clin Lipidol 2017;11:1407-14.

[50] Ruhaak LR, Smit NPM, Romijn FPHTM, et al. Robust and Accurate 2-Year Performance of a Quantitative Mass Spectrometry-Based Apolipoprotein Test in a Clinical Chemistry Laboratory. Clin Chem 2018;64:747-49. [51] Nicolardi S, van der Burgt YE, Dragan I, et al. Identification of new apolipoprotein-CIII glycoforms with ultrahigh resolution MALDI-FTICR mass spectrometry of human sera. J Proteome Res 2013;12:2260-8.

[52] Koska J, Yassine H, Trenchevska O, et al. Disialylated apolipoprotein C-III proteoform is associated with improved lipids in prediabetes and type 2 diabetes. J Lipid Res 2016;57:894-905.

[53] Trenchevska O, Schaab MR, Nelson RW, et al. Development of multiplex mass spectrometric immunoassay for detection and quantification of apolipoproteins C-I, C-II, C-III and their proteoforms. Methods 2015;81:86-92. 
[54] Wolf AM, Wender RC, Etzioni RB, et al. American Cancer Society Prostate Cancer Advisory Committee. American Cancer Society guideline for the early detection of prostate cancer: update 2010. CA Cancer J Clin 2010;60:70-98.

[55] Mottet N, Bellmunt J, Bolla M, et al. EAU-ESTRO-SIOG Guidelines on Prostate Cancer. Part 1: Screening, Diagnosis, and Local Treatment with Curative Intent. Eur Urol 2017;71:618-629.

[56] Kammeijer GSM, Nouta J, de la Rosette J, et al. An In-depth Glycosylation Assay for Urinary Prostate Specific Antigen. Anal Chem 2018 doi: 10.1021/acs.analchem.7b04281.

[57] Wright I, Van Eyk JE. A Roadmap to Successful Clinical Proteomics. Clin Chem 2017;63:245-247. 